\title{
Implementation of Distributed Time Exchange Based Cooperative Forwarding
}

\author{
Muhammad Nazmul Islam, Shantharam Balasubramanian, Narayan B. Mandayam, Ivan Seskar and Sastry \\ Kompella* \\ Wireless Information \& Networking Laboratory, Rutgers University \\ mnislam@winlab.rutgers.edu, shantharampsg@gmail.com, narayan@winlab.rutgers.edu, \\ seskar@winlab.rutgers.edu, * Information Technology Division, Naval Research Laboratory, Email: sk@ieee.org
}

\begin{abstract}
In this paper, we design and implement time exchange (TE) based cooperative forwarding where nodes use transmission time slots as incentives for relaying. We focus on distributed joint time slot exchange and relay selection in the sum goodput maximization of the overall network. We formulate the design objective as a mixed integer nonlinear programming (MINLP) problem and provide a polynomial time distributed solution of the MINLP. We implement the designed algorithm in the software defined radio enabled USRP nodes of the ORBIT indoor wireless testbed. The ORBIT grid is used as a global control plane for exchange of control information between the USRP nodes. Experimental results suggest that TE can significantly increase the sum goodput of the network. We also demonstrate the performance of a goodput optimization algorithm that is proportionally fair.
\end{abstract}

Index Terms-Resource Delegation, Cooperative Forwarding, Global Control Plane, Testbed Implementation, GNUradio.

\section{INTRODUCTION}

Cooperative forwarding improves the connectivity and throughput in wireless networks [1]. However, forwarding always incurs some costs, e.g., delay/power, at the forwarder node. Hence, there have been recent studies on resource delegation based incentivized forwarding [2]-[7]. Resource delegation based forwarding allows the sender node to delegate a portion of its allotted resource to the forwarder node as an immediate incentive for relaying. Previous works on incentivized forwarding have focused on resource exchange from a theoretical perspective and used numerical simulations to justify the effectiveness of the approach. The main contribution of our work is to demonstrate the advantages of incentivized forwarding using the ORBIT indoor wireless testbed [8].

We specifically focus on the uplink of an $N$ node time division multiple access (TDMA) network where each node receives an initial number of time slots and transmits data to the base station (BS) through the direct path. In this context, we focus on a two hop time exchange (TE) based forwarding scheme where the sender node transfers a portion of its allotted time slots to the forwarder node as an incentive for relaying. The basic idea of TE is illustrated in Fig. 1. In this example, node 1 and 2 initially receive 4 time slots and transmit through the direct path. In TE, node 1 performs a half duplex decode and forward (DF) relaying of node 2's data and node 2 delegates one time slot to node 1 as an incentive for relaying. Node 2 may attain higher data rate through this cooperation

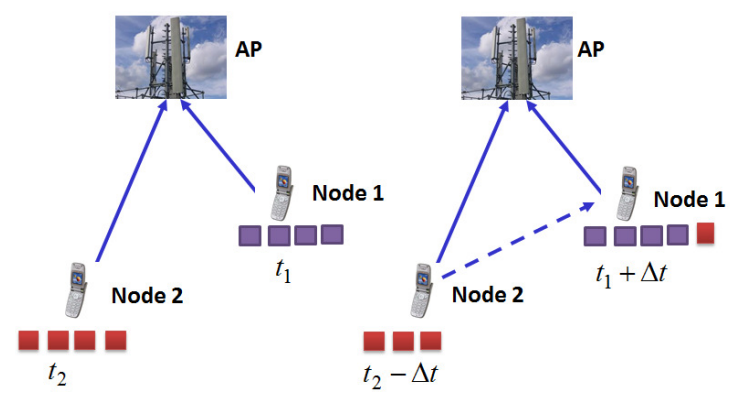

Fig. 1. The motivating scenario for time exchange based cooperative forwarding

since its data goes to the BS through two different paths. Node 1 may also get higher data rate since it has more transmission time slots. The optimal time slot delegation is an important question in this context.

A sender node can delegate time slots to multiple forwarder nodes and transmit data to the BS through multiple paths. However, the authors of [9] have shown that for a sourcedestination pair, in the presence of multiple relay nodes, it is sufficient to select the "best relay node" to achieve full diversity order. On the other hand, the assumption of one sender node for one forwarder node reduces the relay selection complexity. Therefore, we assume one forwarder node for one sender node and vice versa in this work. The optimal distributed sender-forwarder pair selection problem becomes another important question in this context.

In this work, we address the joint time slot delegation and sender-forwarder pair selection question in the context of sum goodput maximization of the overall network. We formulate the joint optimization problem as a mixed integer nonlinear programming (MINLP) problem. Using our relay selection work of [5], we show that the distributed solution of the MINLP requires $O\left(k^{\text {tot }}\right)$ computational complexity and at most $N^{2}$ message passing where $k^{\text {tot }}$ and $N$ denote the total time slots and nodes in the network. The designed algorithm maximizes the sum goodput of the network while preserving the local goodput of the individual nodes.

We implement the designed algorithm in the ORBIT indoor wireless testbed. We use software defined radio enabled USRP [10] nodes of the ORBIT [8] testbed. The ORBIT grid serves as the global coordination plane [11] to exchange the 


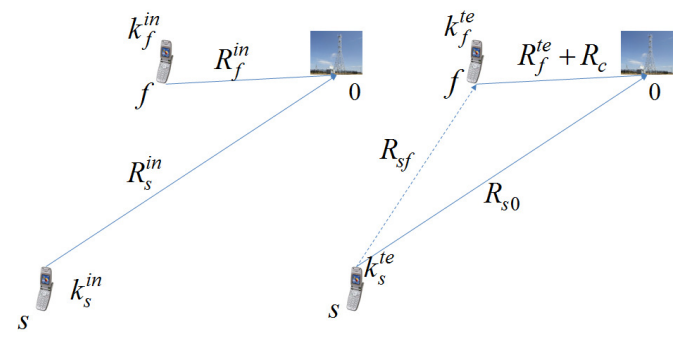

Fig. 2. Direct Transmission and Time Exchange System Model

protocol information between the nodes. The data transmission through the air is processed using the GNUradio codes [12].

Previous works in resource exchange [2]-[7] have focused on developing information theoretical algorithms. We design the theoretical framework in accordance with the testbed constraints and then implement the framework in the ORBIT testbed. Our theoretical analysis has similarities to the classical maximum weighted scheduling study of [13]. However, unlike [13], we consider a network where each node starts with a fixed number of time slots and then tries to find the optimal number of time slot transfers. Our proposed framework can be applied to TDMA based commerical (GSM \& Edge [14], 802.16 Wireless MAN [15]) and tactical (Joint Tactical Radio System [16] and Link16 [17]) networks.

This paper is organized as follows. Sec. II and III illustrate the system model and design objective respectively. We solve the optimization problem in Sec. IV After describing the experimental setup in Sec. V, we demonstrate the testbed results in Sec. VI We conclude the work in VII.

\section{System ModeL}

We consider the uplink of an $N$ node single cell TDMA network. Let $\mathcal{V}=\{1,2, \cdots, N\}$ denote the set of $N$ nodes that transmit data to the BS (node 0). Each node uses the same bandwidth. Node $i \in \mathcal{V}$ is initially allotted $k_{i}^{i n}$ time slots per second. Without loss of generality, assume one packet is transmitted per time slot. Each node is assumed to employ a fixed modulation scheme. This assumption is based on the testbed implementation constraint and will be further explained in the experimental setup section. Due to the fixed modulation and total bandwidth usage, the data transmission rate of each node depends on the number of allotted time slots.

We use packet loss probability as the channel strength indicator. Let $P e_{i j}$ denote the packet loss probability in the $i j$ path. Define goodput of node $i$ by the number of packets of node $i$ that successfully reach the BS. Node $i$ initially transmits through the direct link $i 0$. Hence, the initial goodput of node $i, R_{i}^{i n}$, can be found as:

$$
R_{i}^{i n}=R_{i 0}=k_{i}^{i n} *\left(1-P e_{i 0}\right)
$$

\section{A. Goodput Analysis in TE}

In TE, nodes perform two hop half duplex decode and forward (DF) relaying. Let, $\mathcal{S F}=\left\{\mathcal{S F}_{1}, \cdots, \mathcal{S F}_{k}\right\}=$ $\left\{\left(s_{1}, f_{1}\right),\left(s_{2}, f_{2}\right), \cdots,\left(s_{K}, f_{K}\right)\right\}$ denote the sender-forwarder pairing sets, i.e., the forwarder node $f_{i}$ relays the sender
TABLE I

SUMMARY OF USED NOTATIONS

\begin{tabular}{|l|l|}
\hline Notation & Meaning \\
\hline$P e_{i j}$ & Packer error probability of the $i j$ link \\
\hline$k_{i}^{i n}$ & Initial transmission time slot of node $i$ \\
\hline$k_{i}^{t e}$ & Node $i$ 's btransmission time slot in BE \\
\hline$R_{i}^{i n}$ & Initial goodput of node $i$ \\
\hline$R_{i}^{t e}$ & Node $i$ 's goodput in TE \\
\hline$R_{i j}$ & Goodput in the $i j$ link \\
\hline $\mathcal{V}$ & Set of $N$ nodes \\
\hline $\mathcal{D}$ & Set of nodes that transmit without cooperation \\
\hline $\mathcal{S F}$ & Set of sender-forwarder pairs \\
\hline $\mathcal{S F} \mathcal{F}_{i}$ & $i^{\text {th }}$ sender-forwarder pair $\left(s_{i}, f_{i}\right)$ \\
\hline$s$ & Sender node \\
\hline$f$ & Forwarder Node \\
\hline$k^{t o t}$ & Total time slots in 1 second \\
\hline$n_{i}$ & Number of neighbouring nodes of node $i$ \\
\hline
\end{tabular}

node $s_{i}$ 's data, along with transmitting $f_{i}$ 's own data. Let $\mathcal{D}=d_{1}, d_{2}, \cdots, d_{L}$ denotes the direct set, i.e., the set of remaining nodes that transmit data without cooperation.

The left and right hand side of Fig. 2 shows the direct transmission and TE model of an arbitrary sender-forwarder pair $(s, f)$. Node $s$ and $f$ initially receive $k_{s}^{i n}$ and $k_{f}^{i n}$ time slots and obtain $R_{s}^{i n}$ and $R_{f}^{i n}$ goodput respectively.

In TE, node $s$ transmits for $k_{s}^{t e}$ time slots. During $k_{s}^{t e}$ time slots, node $s$ transmits $k_{s}^{t e}$ packets. Among these packets, the BS and node $f$ receive $R_{s 0}$ and $R_{s f}$ 'error-free' packets respectively. Following the analysis of (1),

$$
R_{s f}=k_{s}^{t e} *\left(1-P e_{s f}\right), R_{s 0}=k_{s}^{t e} *\left(1-P e_{s 0}\right)
$$

Assume $P e_{s f} \leq P e_{s 0}$. Hence, $R_{s f} \geq R_{f 0}$. Node $f$ acts as a forwarder of node $s$ and transmits for $k_{f}^{t e}$ time slots. During these time slots, $k_{f}^{t e} *\left(1-P e_{f 0}\right)$ packets 'successfully' reach the BS. Assume,

$$
R_{f}^{t e}+R_{c}=k_{f}^{t e} *\left(1-P e_{f 0}\right)
$$

Here, $R_{f}^{t e}$ denotes the number of 'error-free' packets that contain node $f$ 's own data. $R_{c}$ represents the number of 'errorfree' packets that contain node $s$ 's data and are forwarded by node $f$.

Let $R_{s}^{t e}$ denote the goodput of node $s$ in TE. Also assume that when node $s$ transmits, the packets that get 'lost' at node $f$ (the closer node), also get 'lost' at node 0 (the far node). Based on this assumption and using the max-flow-min-cut theorem [18],

$$
R_{s}^{t e} \leq \min \left(R_{s f}, R_{s 0}+R_{c}\right)
$$

Based on this goodput analysis, we focus on the distributed joint optimal time slot exchange and relay selection in the sum goodput maximization of a TE network.

\section{Design ObJective}

Problem I

$$
\max \cdot \sum_{d \in \mathcal{D}} R_{d}^{t e}+\sum_{(s, f) \in \mathcal{S F}}\left(R_{f}^{t e}+R_{s}^{t e}\right)
$$




$$
\begin{gathered}
\text { s.t. }\left(R_{f}^{t e}, R_{s}^{t e}\right) \in \operatorname{conv}\left(k_{f}^{t e}, k_{s}^{t e}\right) \forall(s, f) \in \mathcal{S F} \\
R_{f}^{t e} \geq R_{f}^{i n}, R_{s}^{t e} \geq R_{s}^{i n} \forall(s, f) \in \mathcal{S F} \\
k_{f}^{t e}+k_{s}^{t e} \leq k_{f}^{i n}+k_{s}^{i n},\left(k_{f}^{t e}, k_{s}^{t e}\right) \in \mathcal{Z}^{+}, \forall(s, f) \in \mathcal{S F} \\
R_{d}^{t e}=k_{d}^{t e} *\left(1-P e_{d 0}\right), k_{d}^{t e}=k_{d}^{i n}, \forall d \in \mathcal{D} \\
\mathcal{D} \subseteq \mathcal{V}, \mathcal{S F} \in \mathcal{V} \times \mathcal{V}, \mathcal{S F}_{i} \cap \mathcal{S F}_{j}=\emptyset \forall i \neq j \\
\mathcal{S} \mathcal{F}_{i} \cap \mathcal{D}=\emptyset \forall i \in[1, K] \\
\mathcal{S} \mathcal{F}_{1} \cup \mathcal{S} \mathcal{F}_{2} \cdots \cup \mathcal{S} \mathcal{F}_{K} \cup \mathcal{D}=\mathcal{V}
\end{gathered}
$$

$$
\text { Variables } \mathcal{D}, \mathcal{S F}, R_{s}^{t e}, R_{f}^{t e}, k_{s}^{t e}, k_{f}^{t e}
$$

Equation (5e) and (1) suggest that $R_{d}^{t e}=R_{d}^{i n}$. Therefore, the goodputs of the nodes in the direct set $\mathcal{D}$ are not optimization variables. Equation $(5 \mathrm{~b})$ denotes that the goodputs of the forwarder and sender remain in the convex hull of the allotted time slots, $k_{s}^{t e}$ and $k_{f}^{t e}$. This convex hull is governed by (2) and (3). Equation (5c) ensures that the goodputs of the sender and the forwarder through TE don't drop below their initial goodputs. Equation (5d) shows that the total time slots used by the sender and forwarder are constrained by the summation of the initial time slots allotted to those nodes. Equation (5f)(5h) denote that the direct node set and the sender-forwarder pairs cannot have any common node and together, they form the overall set $\mathcal{V}$.

Problem I is similar to the design objective of our earlier work on bandwidth exchange [5]. However, we focused on information theoretic capacity [19] based resource allocation and relay selection in [5]. In this work, we focus on packet error probability based goodput maximization, which is a tangible objective in an indoor wireless testbed.

The convex constraint of (3), the discrete time slot allocation and the sender-forwarder pair selection objectives make problem I a mixed integer nonlinear programming (MINLP) problem. The solution of this MINLP involves an exponential number of variables and constraints. In the next section, we focus on designing a polynomial time distributed solution of problem I.

\section{Optimization Problem Solution}

Let $R_{t o t}=\sum_{i \in \mathcal{V}} R_{i}^{i n}$ denote the summation of the initial goodputs of the nodes. For a fixed $\mathcal{S} \mathcal{F}, R_{t o t}$ can be expressed in the following form:

$$
\begin{aligned}
R_{\text {tot }} & =\sum_{i \in \mathcal{V}} R_{i}^{i n} \\
& =\sum_{d \in \mathcal{D}} R_{d}^{i n}+\sum_{(s, f) \in \mathcal{S F}}\left(R_{f}^{i n}+R_{s}^{i n}\right) \\
& =\sum_{d \in \mathcal{D}} R_{d}^{t e}+\sum_{(s, f) \in \mathcal{S} \mathcal{F}}\left(R_{f}^{i n}+R_{s}^{i n}\right)
\end{aligned}
$$

Equation (7) uses the fact that $R_{d}^{t e}=R_{d}^{i n} \forall d \in D$. Subtracting $R_{t o t}$ from the objective function of I, we find the following optimization problem:

Problem II

$$
\begin{gathered}
\max . \sum_{(s, f) \in \mathcal{S} \mathcal{F}}\left(R_{f}^{t e}+R_{s}^{t e}-R_{f}^{i n}-R_{s}^{i n}\right) \\
\text { s.t. }\left(R_{f}^{t e}, R_{s}^{t e}\right) \in \operatorname{conv}\left(k_{f}^{t e}, k_{s}^{t e}\right) \forall(s, f) \in \mathcal{S F} \\
R_{f}^{t e} \geq R_{f}^{i n}, R_{s}^{t e} \geq R_{s}^{i n} \forall(s, f) \in \mathcal{S F} \\
k_{f}^{t e}+k_{s}^{t e} \leq k_{f}^{i n}+k_{s}^{i n},\left(k_{f}^{t e}, k_{s}^{t e}\right) \in \mathcal{Z}^{+}, \forall(s, f) \in \mathcal{S F}
\end{gathered}
$$

$$
\begin{gathered}
\mathcal{D} \subseteq \mathcal{V}, \mathcal{S F} \in \mathcal{V} \times \mathcal{V}, \mathcal{S F}_{i} \cap \mathcal{S F}_{j}=\emptyset \forall i \neq j \\
\mathcal{S F}_{i} \cap \mathcal{D}=\emptyset \forall i \in[1, K] \\
\mathcal{S F}_{1} \cup \mathcal{S F}_{2} \cdots \cup \mathcal{S F}_{K} \cup \mathcal{D}=\mathcal{V}
\end{gathered}
$$

$$
\text { Variables } \mathcal{D}, \mathcal{S F}, R_{s}^{t e}, R_{f}^{t e}, k_{s}^{t e}, k_{f}^{t e}
$$

The inclusion of constant terms does not change the optimal variables of an optimization problem [20]. Therefore, the optimal variables of both problem I and II are same. We focus on solving problem II in the subsequent analysis and use the optimal variables to find the solution of problem I.

Problem II involves both sender-forwarder pair selection and discrete time slot allocation features. For a fixed set of senderforwarder pairs, the constraints in $8 \mathrm{~b}$ - $8 \mathrm{~d}$ ) ensure that the discrete time slot exchange in one pair does not affect the other pairs. Hence, we now focus on an arbitrary sender-forwarder pair $(s, f)$ and find the discrete time slot exchange in this pair.

\section{A. Time Slot Allocation for a Fixed Sender Forwarder Pair}

\section{Problem III}

$$
\max \left(R_{s}^{t e}-R_{s}^{i n}\right)+\left(R_{f}^{t e}-R_{f}^{i n}\right)
$$

$$
R_{s f}=k_{s}^{t e} *\left(1-P e_{s f}\right), R_{s 0}=k_{s}^{t e} *\left(1-P e_{s 0}\right)
$$

$$
R_{f}^{t e}+R_{c}=k_{f}^{t e} *\left(1-P e_{f 0}\right), R_{s}^{t e} \leq \min \left(R_{s f}, R_{s 0}+R_{c}\right)
$$




$$
\begin{gathered}
R_{f}^{t e} \geq R_{f}^{i n}, R_{s}^{t e} \geq R_{s}^{i n} \\
k_{f}^{t e}+k_{s}^{t e} \leq k_{f}^{i n}+k_{s}^{i n},\left(k_{f}^{t e}, k_{s}^{t e}\right) \in \mathcal{Z}^{+} \\
\text {variables } R_{c}, R_{s f}, R_{s 0}, R_{s}^{t e}, R_{f}^{t e}, k_{s}^{t e}, k_{f}^{t e}
\end{gathered}
$$

Lemma 2: Problem III is concave if $\left(k_{f}^{t e}, k_{s}^{t e}\right) \in \mathcal{R}^{+}$.

Proof: The objective function in (9a) and the constraints in $(9 \mathrm{~b}),(9 \mathrm{c})$ and $(9 \mathrm{e}$ ) are linear. Minimum of linear (concave) functions is concave [20]. Hence, the constraint in $9 \mathrm{c}$ is convex. Thus, problem III is a concave maximization problem if $\left(k_{f}^{t e}, k_{s}^{t e}\right) \in \mathcal{R}^{+}$.

The internal concave structure of problem III allows us to generate an upper and lower bound of problem III.

1) Upper Bound: Let us modify problem III by relaxing the integer time slot constraint, i.e., let us assume $\left(k_{f}^{t e}, k_{s}^{t e}\right) \in$ $\mathcal{R}^{+}$. Let's call it problem IV. Now, the feasible region of the modified problem is a superset of that of problem III. Hence, the optimal solution of the modified problem is an upper bound of problem III. Denote this upper bound by $u^{0}$.

2) Lower Bound: Let $k_{s}^{t e, *}$ and $k_{f}^{t e, *}$ denote the optimal time slot solutions of problem IV. Now, if $k_{s}^{t e, *}$ and $k_{f}^{t e, *}$ are integers, they are also the optimal solutions of problem III. Otherwise, convert $k_{s}^{t e, *}$ and $k_{f}^{t e, *}$ to the nearest integers and find the corresponding goodputs. If the corresponding solution is feasible for problem III, it can serve as a lower bound to the optimal solution of problem III. Denote this lower bound by $l^{0}$.

3) Computational Complexity of Problem III: Probelm III can be optimally solved by searching over $k_{s}^{i n}$ time slot transfers. However, one can further reduce the complexity by solving the convex programming based upper and lower bounds of problem III. Let $\epsilon$ denote the tolerance of the optimal solution. If $\left(u_{0}-l_{0}\right) \leq \epsilon$, we can use the lower bound as the solution of problem III.

\section{B. Optimal Sender Forwarder Pair Selection}

The optimal solution of problem III denotes the senderforwarder pair's goodput gain through cooperation, over noncooperation. The optimal relay selection part of problem II is to find the set of sender-forwarder pairs that maximizes the summation of the goodput gain. Now, consider a graph $\mathcal{G}=(\mathcal{V}, \mathcal{E})$ where the vertices $\mathcal{V}$ represent the set of $N$ nodes under consideration and $\mathcal{E}$ denotes the edges between these nodes. Define the edge weight of any $(i, j)$ pair by $R_{i}^{t e}+R_{j}^{t e}-R_{i}^{i n}-R_{j}^{i n}$, i.e., the difference, in terms of goodput, between the cooperation and non-cooperation scenario. Using the interference-free scheduling algorithm of [13], the optimal sender-forwarder set selection problem can be shown to be equivalent to solving the maximum weighted matching (MWM) algorithm [21] in the above graph. A detailed proof of the equivalence between the relay selection problem and the MWM algorithm can be found in [5]. The next section will illustrate the use of MWM in the optimal sender-forwarder pair selection among 3 testbed nodes.

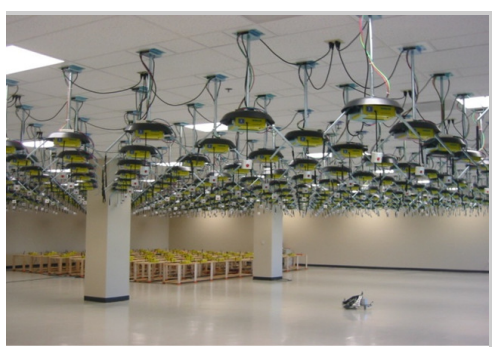

Fig. 3. Orbit Testbed

The MWM algorithm can be distributively solved using the distributed local greedy MWM [22]. Distributed MWM finds the pairs by selecting the locally heaviest edges and guarantees at least $50 \%$ performance of the optimal solution of problem I [22]. Our distributed TE implementation protocol is based on the distributed MWM and will be described in the next section.

\section{Distributed TE Protocol}

1) Node $i \in \mathcal{V}$ has $k_{i}^{i n}$ transmission time slots and knows the packet error probability of its direct path, $P e_{i 0}$,

2) Let $\mathcal{J}$ be the set of neighbours of $i$. In a wireless environment, neighbouring nodes can hear each other. Node $j \in \mathcal{J}$ receives node $i$ 's packets and can calculate the inter-node packet error probability, $P e_{i j}$.

3) Node $i$ sends an omnidirectional message containing $P e_{i 0}$ and $k_{i}^{i n}$ to its neighbours.

4) Node $i$ solves problem III for all $j \in \mathcal{J}$ and finds the goodput gain for each of its neighbours. Thus, node $i$ knows its adjacent link weights.

5) Node $i$ picks the "candidate" node $j$, based on the heaviest adjacent link weight and sends "add" request.

6 ) If node $i$ receives an "add" request from node $j, i$ and $j$ form a cooperative pair. Node $i$ sends "drops" request to its other neighbours.

7) If node $i$ receives a "drop" request from $j$, node $i$ removes the $(i, j)$ link from its adjacent edge set. Node $i$ returns to step 5 .

8) The pair selection process converges after at most $N^{2}$ message passings. The 'matched' nodes form the set of cooperative pairs $\mathcal{S} \mathcal{F}$. The 'unmatched' nodes form $\mathcal{D}$ and transmit data without cooperation.

9) The sender node $s$ of a cooperative pair transmits its own data during $k_{s}^{\text {te }}$ time slots.

10) The BS sends ACK of the 'correctly' received packets to the sender node. The forwarder node also hears these ACK messages. Based on this information, node $f$ finds the packets of $s$ that got 'lost' at the BS.

11) Node $f$ transmits for $k_{f}^{t e}$ time slots. During these slots, node $f$ forwards the 'lost' packets of $s$ and then transmits its own data packets to the BS.

\section{Computational Complexity of the Distributed Algorithm}

Each node $i \in \mathcal{V}$ solves problem III for each of its neighbours. Let $n_{i}$ be the number of neighbours of node 


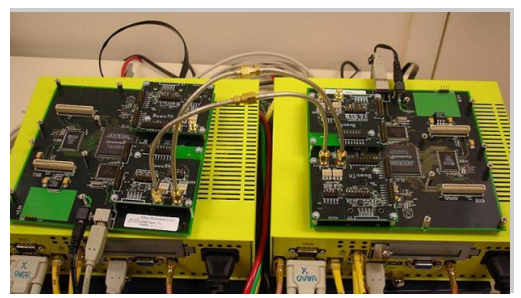

Fig. 4. USRP Daughterboards

$i$. Since problem III can be optimally solved using $O\left(k_{i}^{i n}\right)$ searches, each node performs $O\left(n_{i} k_{i}^{i n}\right)$ computations. The total number of computations in the $N$ node network is $O\left(n_{i} k_{i}^{i n} N\right)$.

The number of time slots allotted to node $i, k_{i}^{i n}$, can be approximated as, $k_{i}^{i n} \approx \frac{k^{t o t}}{N}$. Since $n_{i}<<k^{\text {tot }}$, the overall complexity is, $O\left(k^{t o t}\right)$.

Thus, the proposed TE protocol solves an approximated distributed version of problem I with $O\left(k^{t o t}\right)$ complexity and at most $N^{2}$ message passings.

\section{EXPERIMENTAL SETUP}

\section{A. ORBIT Testbed \& USRP Nodes}

We implement the proposed TE based incentivized algorithm among the USRP nodes of ORBIT, an indoor wireless testbed of Wireless Information Network Laboratory (WINLAB), Rutgers University. ORBIT has 400 nodes, overall, in a $20 m \times 20 m$ square grid. Fig. 3 shows a snapshot of the ORBIT testbed.

ORBIT has 15 USRP nodes that can be used in software defined radio based experiments. Fig. 4 shows the snapshots of two USRP daughter boards. We use the GNU radio software toolkil [12] to run experiments in these USRP nodes. Specifically, we use the benchmark-tx.py and benchmarkrx.py codes to transmit and receive packets between two USRP nodes [12]. The flexibility of GNUradio allows us to change the transmission power level and packet sizes through software. This variable power capability of GNUradio, along with the spatial separation among the nodes, allow us to create links with different strengths between different node pairs.

As shown in Fig. 5, we use four USRP nodes of the ORBIT testbed to conduct the TE based cooperative forwarding experiments. Fig. 5 also shows the spatial separation of the selected nodes. Here, node 1,2 and 3 constitute the user set $\mathcal{V}$ and node 0 serves as the BS. The ORBIT grid is used as a global control plane to exchange the control information between the nodes.

\section{B. Selection of Parameters}

The benchmark-tx.py and benchmark-rx.py codes of GNUradio allow the following four modulation schemes: a) GMSK, b) differential binary phase shift keying (DBPSK), c) differential quadrature phase shift keying (DQPSK) and d) differential 8 phase shift keying (D8PSK). DBPSK, DQPSK and D8PSK are found to be very sensitive to peak power clippings due to their variable envelope waveform. Therefore, we use a fixed modulation scheme, GMSK, in our experiments.

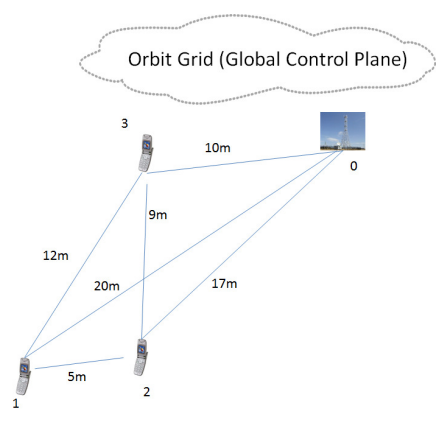

Fig. 5. Spatial Separation of Selected Nodes
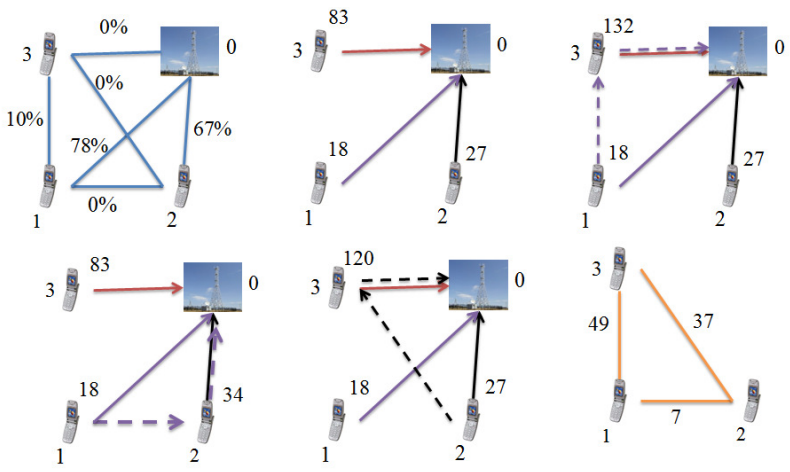

Fig. 6. Illustration of MWM in sender-forwarder pair selection

Each node transmits at $1 \mathrm{Mbps}$ and each packet contains 1500 bytes. As a result, it takes $(1500 * 8) /\left(1 * 10^{6}\right)$, i.e., 0.012 second to transmit one packet. We assume each time slot to be 0.012 second long, i.e., one packet is transmitted in each slot.

The total transmission time is assumed to be 3 second. Each node is initially allotted 1 second transmission time, i.e., $1 / 0.012$ or 83 time slots. We approximate the number of time slots since fractional packet transmission is not considered.

We also add 32 bit CRC sequence in each packet and make it similar to the Ethernet packet structure [23]. Note that, we do not use error control coding in these experiments. Therefore, the presence of a single bit error leads to the 'loss' of the whole packet due to CRC.

\section{EXPERIMENTAL EVALUATION}

\section{A. Illustration of MWM in Relay Selection}

Fig. 6 shows the use of MWM in the optimal senderforwarder pair selection among 3 testbed nodes. The left figure of the top row shows the packet loss probability between the inter-node pairs. These packet error probabilities were based on 1500 byte packet length, GMSK modulation, some fixed power level and CRC checking. The middle figure of the top row focuses on the direct transmission scenario and shows the goodput (in packet/3 second) of each node. Each node initially receives 83 time slots and transmits one packet at each slot through the direct path. The packet error probability in link 30 is $0 \%$. Therefore, all transmitted packets of node 3 reach the BS. Node 1 and 2's goodputs are considerably lower due to the high packet error probability in link 10 and 20 respectively. 


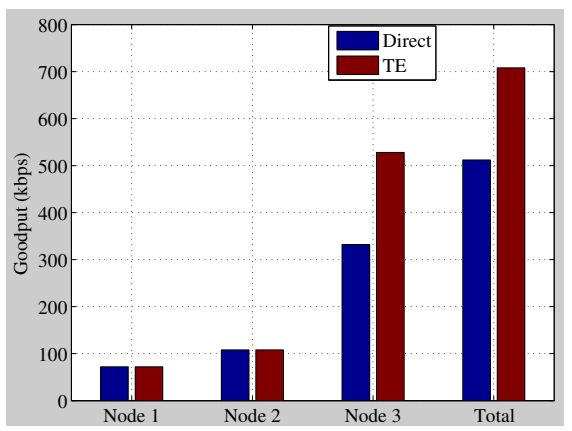

Fig. 7. Sum Goodput Maximization in 3 node (Packet length $=1500$ bytes, CRC checking, GMSK modulation)

The top right, the bottom left and the bottom middle figure show the goodput (in packet/ 3 second) of different senderforwarder cooperation scenarios. The top right figure focuses on the TE based cooperation between node 1 and 3 . Here, node 1 and 3 solve the two node time slot allocation optimization of problem III. The cooperation allows node 3 to achieve a goodput of 132 packets and ensures that node 1's goodput does not drop below 18 packets, its initial value. Therefore, the overall goodput gain obtained through the cooperation of node 1 and 3 is 49 packets. As a result, the 13 link of the MWM graph, shown in the bottom right figure, is assigned a weight of 49 .

The bottom left and bottom middle figures demonstrate the cooperation scenario in node 1-2 and 2-3 respectively. The bottom right figure shows the link weight of the corresponding cooperation pairs. The distributed local greedy MWM selects link 13. Therefore, node 1 and 3 cooperate using TE, whereas, node 2 transmits without cooperation.

\section{B. Sum Goodput Maximization}

Fig. 7 compares the sum goodput (in kilo bit per second (kbps)) of TE and direct path transmission. Fig. 6 shows that node 1 and 3 get selected as the cooperative pair due to the MWM algorithm. Therefore, node 1 and 3 solve problem III to find the optimal time slot transfers. Due to the sum goodput maximization objective, the benefits of cooperation go to node 3 , i.e., the node with the better channel. Node 3's goodput increases by $70 \%$. The constraint of 9d ensures that node 1 gets its initial goodput, at least. On the other hand, node 2 transmits without cooperation and its goodput does not change from the initial value.

\section{Proportional Fair Maximization of Goodput}

Fig. 8 compares the proportional fair maximization performance of direct transmission and TE. Here, the selected cooperative nodes, $s$ and $f$, solve a modified version of problem III. In this modified problem, $s$ and $f$ maximize $\left(R_{s}^{t e}-R_{s}^{i n}\right) *\left(R_{f}^{t e}-R_{f}^{i n}\right)$ instead of maximizing $\left(R_{s}^{t e}+R_{f}^{t e}\right)$. Hence, the goodput of both nodes increase due to cooperation. Fig. 8 shows that the goodputs of node 1 and 3 increase by $70 \%$ and $30 \%$ respectively.

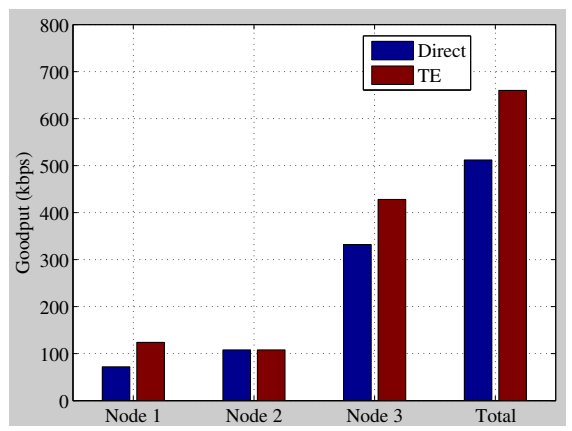

Fig. 8. Proportional Fair Maximization of Goodput (Packet length $=1500$ bytes, CRC checking, GMSK modulation)

\section{CONCLUSION}

We designed and implemented TE based cooperative forwarding among the USRP nodes in the ORBIT indoor wireless testbed. We solved the joint time slot allocation and senderforwarder pair selection problem in this setup. Our proposed algorithm maximizes the global goodput of the network while ensuring that no node's goodput drops below its initial value. The ORBIT grid is used as a global control plane to exchange the control information between the USRP nodes. Experimental results suggest that resource delegation based cooperative forwarding can significantly improve the sum goodput and proportional fair goodput performance of the network.

The use of adaptive modulation and signal to noise ratio based resource allocation in testbed implementation remains an area of future research.

\section{ACKNOWLEDGEMENTS}

This work is supported by the Office of Naval Research under grant N00014-11-1-0132. We thank Kush Patel, Sid Paradkar and Hakim Ergaibi for their assistance in GNUradio coding and testbed implementation.

\section{REFERENCES}

[1] J. N. Laneman, D. N. C. Tse, and G. Wornell, "Cooperative diversity in wireless networks : efficient protocols and outage behavior," IEEE Trans. Info. Theory, vol. 50(12), pp. 3062-3080, Dec. 2004.

[2] D. Zhang, R. Shinkuma, and N. B. Mandayam, "Bandwidth exchange: An energy conserving incentive mechanism for cooperation," IEEE Trans. Wireless Comm, vol. 9(6), pp. 2055-2065, June 2010.

[3] H. Xu and B. Li, "Efficient resource allocation with flexible channel cooperation in OFDMA cognitive radio networks," in Proc. IEEE INFOCOM'2010, Mar. 2010, pp. 1-9.

[4] Z. Han, T. Himson, W. P. Siriwongpairat, and K. J. Ray Liu, "Resource allocation for multiuser cooperative ofdm networks: Who helps whom and how to cooperate," IEEE Trans. Vehicular Technology, vol. 58(5), pp. 2378-2391, June 2009.

[5] M. N. Islam, N. B. Mandayam, and S. Kompella, "Optimal resource allocation and relay selection in bandwidth exchange based cooperative forwarding," To Appear in WiOpt 2012, Jan 2012, http://arxiv.org/abs/1112.5767.

[6] J. Zhang and Q. Zhang, "Stackelberg game for utility-based cooperative cognitive radio networks," in Proc. ACM MOBIHOC'2009, May 2009, pp. 23-31.

[7] M. Lindstorm and P. Lungaro, "Resource delegation and rewards to stimulate forwarding in multihop cellular networks," in Proc. IEEE VTC'2005, June 2005.

[8] "ORBIT: Open access research testbed for next-generation wireless networks," accessed April 2012, http://www.orbit-lab.org. 
[9] Y. Zhao, R. Adve, and T. J. Lim, "Improving amplify-and-forward relay networks: Optimal power allocation versus selection," IEEE Trans. Wireless Comm, vol. 6(8), pp. 3114-3123, Aug. 2007.

[10] "ETTUS research," accessed April 2012, http://www.ettus.com.

[11] D. Raychaudhuri, N. B. Mandayam, J. B. Evans, B. J. Ewy, S. Seshan, and P. Steenkiste, "CogNet: an architectural foundation for experimental cognitive radio networks within the future internet," in Proc. MobiArch'2006, Dec. 2006.

[12] "GNU Radio Website," accessed April 2012, http://www.gnuradio.org.

[13] L. Tassiulas and A. Ephremides, "Stability properties of constrained queing systems and scheduling for maximum throughput in multihop radio networks," IEEE Trans. Automatic Control, vol. 37(12), pp. 19361949, Dec. 1992.

[14] A. Goldsmith, Wireless Communications, Cambridge University Press, New York, NY, 2005.

[15] C. Eklund, R. B. Marks, K. L. Stanwood, and S. Wang, "IEEE standard 802.16: a technical overview of the WirelessMAN/sup TM/ air interface for broadband wireless access," IEEE Communications Magazine, vol. 40, pp. 98-107, June 2002.

[16] C. D. Young, "The mobile data link (MDL) of the joint tactical radio system wideband networking waveform," in Proc. IEEE MILCOM 2007, Oct. 2006.

[17] Air Land Sea Application Center, Fortmonroe, VA, Introduction to Tactical Digital Information Link J and Quick Reference Guide, 2000.

[18] T. Cover and H. El Gamal, "Capacity theorems for the relay channel," IEEE Trans. Info. Theory, vol. 25(5), pp. 572-584, Sept. 1979.

[19] T. M. Cover and J. A. Thomas, Elements of Information Theory, John Wiley and Sons, Hoboken, NJ, 2005.

[20] S. Boyd and L. Vandenberghe, Convex Optimization, Cambridge University, Cambridge, UK, 2004.

[21] J. Edmonds, "Paths, trees and flowers," Canadian Journal of Mathematics, vol. 17, pp. 449-467, 1965.

[22] R. Preis, "Linear time 1/2-approximation algorithm for maximum weighted matching in general graphs," in General Graphs, Symposium on Theoretical Aspects of Computer Science, STACS 99. 1998, pp. 259269 , Springer.

[23] "Ethernet frame," accessed April 2012, http://www.infocellar.com/networks/ethernet/frame.htmg. 